\title{
Palmar Hand Interosseus Muscle
}

National Cancer Institute

\section{Source}

National Cancer Institute. Palmar Hand Interosseus Muscle. NCI Thesaurus. Code C52704.

Three small muscles, located on the second, fourth, and fifth fingers, on the palmar surfaces of the metacarpal bones facilitating the adduction, flexion and extension of the fingers. 